\title{
Hemipelvektomie: Worauf kommt es an?
}

\author{
Markus Nottrott, Arne Streitbürger, Georg Gosheger, Ralf Dieckmann, Carsten Gebert, \\ Marcel-Philipp Henrichs, Jendrik Hardes
}

\section{Zusammenfassung}

Bei primär malignen Knochentumoren des Beckens ist die Hemipelvektomie oft das chirurgische Vorgehen der Wahl neben Polychemotherapie und Radiatio (bei Ewing-Sarkomen). Die Hemipelvektomie ist entweder intern (extremitätenerhaltend) oder extern möglich. Die externe Hemipelvektomie ist zumeist bei Tumorbefall von Glutealmuskulatur oder bei einer großen intrapelvinen Tumorkomponente indiziert. Inoperabilität besteht zumeist nur bei Mittellinienüberschreitung des Sakrums und Multifokalität. Eine komplette Sakrektomie ist möglich, aber äußerst mutilierend. Die Hemipelvektomie ist ein komplikationsreicher Eingriff, der aber oftmals die einzige kurative Möglichkeit für den Patienten darstellt. Postoperative Funktionsdefizite sind unumgänglich und müssen präoperativ individuell besprochen werden. Die Patienten sind aber zumeist trotz Funktionsdefizit zufrieden.

\section{Hemipelvectomy: What is Important?}

Hemipelvectomy is often the therapy of choice, besides polychemotherapy and radiotherapy (for Ewing's sarcoma), for primary malignant pelvic bone tumors. The procedure can be performed either internally (limb-preserving) or externally. An external hemipelvectomy is mainly indicated when the tumor infiltrates the gluteal muscles, making a soft tissue coverage impossible, or when large intrapelvic tumours infiltrate the vessels. Multifocality or crossing the midline of the sacrum are contraindications for a hemipelvectomy. A total sacrectomy would be possible but is extremely mutilating. In spite of being at high risk for complications, the hemipelvectomy is often the only chance for the patient to be cured. The postoperative loss of function has to be critically discussed with the patient preoperatively. Thus, most of the patients are satisfied with the results in spite of the functional deficits.

\section{Einleitung}

Die Hemipelvektomie, also die Resektion von Teilen des knöchernen Beckens, stellt die operative Therapie der Wahl bei primär malignen Knochentumoren des Beckens dar. Aufgrund der Seltenheit von primär malignen Knochentumoren (Inzidenz 4-5/Mio. Einwohner) im Allgemeinen und von Beckentumoren im Speziellen ist dies ein sehr selten durchgeführtes operatives Verfahren. Die Therapie sollte daher in spezialisierten Zentren erfolgen, die eine enge Zusammenarbeit zwischen

OP-JOURNAL 2010; 26: 192-195

(c) Georg Thieme Verlag KG Stuttgart · New York DOI http://dx.doi.org/10.1055/s-0030-1250544
Onkologen, Strahlentherapeuten, Anästhesisten und spezialisierten Operateuren (Orthopäde, plastischer Chirurg, Gefäßchirurg, Neurochirurg) gewährleisten können.

Die Operation hat primär das Ziel, das Überleben des Patienten zu sichern. Erst sekundär kommt der Wiederherstellung der Funktion und des kosmetischen Aspekts eine Bedeutung zu und dies muss dem Patienten im präoperativen Aufklärungsgespräch auch sehr deutlich vermittelt werden.

Es ist zu unterscheiden zwischen interner, extremitätenerhaltender und externer Hemipelvektomie, die mit einem Verlust der Extremität einhergeht. Auf- grund der anatomischen Nähe zwischen Tumor und vitalen Gefäß- und Nervenbahnen im Becken ist die Hemipelvektomie ein höchst anspruchsvoller operativer Eingriff, der einer umfassenden präoperativen Planung bedarf.

\section{Präoperative Planung}

Eine eindeutige histopathologische Diagnose ist für die Durchführung einer Hemipelvektomie zwingend erforderlich. Die hierfür notwendige Biopsie muss schon als Teil der endgültigen operativen Versorgung gesehen werden und sollte daher von dem Operateur durchgeführt werden, der auch die endgültige operative Versorgung durchführen wird. Dies, um einen bioptischen Zugangsweg zu wählen, der im Verlauf des endgültigen operativen Zugangswegs liegt, um so unnötige Kontamination von Gewebe zu vermeiden. Aufgrund eines falsch gewählten bioptischen Zugangswegs kann eine operative Versorgung mit dem Ziel der Tumorfreiheit unmöglich gemacht werden (siehe auch den Beitrag von Henrichs et al. in dieser Ausgabe).

Ein Patient ist deshalb mit einem Beckentumor umgehend an die Institution zu überweisen, die die endgültige Versorgung vornehmen soll.

Eine sorgfältige Indikationsstellung sowie eine gute interdisziplinäre präoperative Planung sind zur Vermeidung von Komplikationen essenziell. Eine standardisierte Bildgebung ist notwendig, um die lokale Tumorausdehnung und das Krankheitsstadium bzw. die Gesamtprognose korrekt erfassen zu können (Staging). Dieses Staging beinhaltet konventionelle Röntgenbilder, CT, MRT und nuklearmedizinische Verfahren (Skelettszintigrafie und PET-CT). Abhängig vom Staging kann man entscheiden, ob eine völlige Tumorfreiheit postoperativ erreicht werden kann. Sollte dies nicht möglich sein, ist die Indikation zur Hemipelvektomie sehr kritisch zu stellen 
und nur noch aus palliativen Gründen bei z.B. drohender Exulzeration indiziert.

Die Entscheidung zur Hemipelvektomie muss immer individuell in enger Zusammenarbeit mit dem Patienten in einem interdiziplinären Forum getroffen werden.

Sehr große Tumoren, die die Mittellinie überschreiten oder multifokale Tumoren können inoperabel sein, da das Ziel einer weiten Tumorresektion in kurativer Absicht nicht erreicht werden kann. Als Ausnahmeindikation sei hier die Metastasenchirurgie (solitäre Spätmetastase der Niere) erwähnt, bei der ebenfalls eine langfristige Tumorfreiheit trotz metastasierendem Krankheitsbild zu erreichen ist.

Die weite Tumorresektion, also das Entfernen des Tumors im gesunden Gewebe, ist im Becken oftmals nur schwer zu erreichen, da die anatomische Nähe $\mathrm{zu}$ vitalen Gefäß-Nerven-Bahnen als limitierender Faktor häufig nur eine marginale Resektion zulässt, jedoch stellt dies häufig die einzige Möglichkeit für den Patienten dar, seine Primärerkrankung zu überleben.

Als Besonderheit ist die Möglichkeit der alleinigen Chemo-Strahlentherapie bei Ewing-Sarkomen zu erwähnen, wenn die Hemipelvektomie zu mutilierend wäre.

\section{OP-Vorbereitung}

In der direkten OP-Vorbereitung sollte am Vortag der Operation eine Darmreinigung durchgeführt werden.

Am OP-Tag ist neben dem üblichen anästhesiologischen Monitoring insbesondere darauf zu achten, dass genügend Blutprodukte (15-20 Blutkonserven, Thrombozytenkonzentrate, FFP) zur Verfügung stehen.

Nach Einleitung der Narkose sollte eine Ureterschienung der betroffenen Seite durchgeführt werden, um so intraoperativ durch eine bessere Identifizierung des Ureters eine iatrogene Schädigung zu vermeiden.

\section{Einteilung der Beckenresektionen}

Abhängig von der Tumorlokalisation im Becken teilt man die $\mathrm{zu}$ resezierenden Anteile des knöchernen Beckens nach

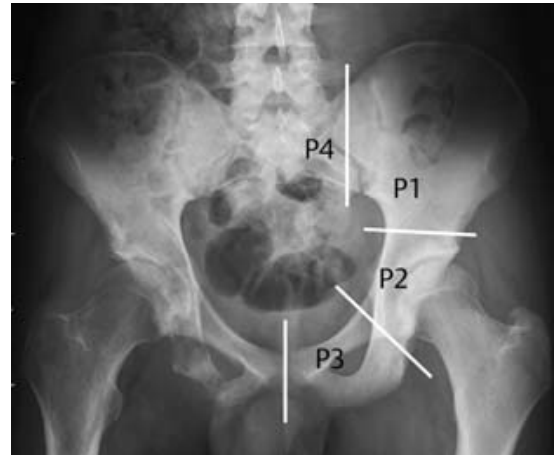

Abb. 1 Einteilung der Beckenresektion nach Enneking: P1 Os ilium, P2 Azetabulum, P3 Os pubis und ischium, P4 Sakrum.

Enneking in 4 Abschnitte (P1-4) ein [2] (Abb. 1).

Bei der P1-Resektion wird das Os ilium vom Iliosakralgelenk bis oberhalb des Azetabulums entfernt. Man unterscheidet eine P1a-Resktion ohne Durchtrennung der knöchernen Kontinuität des Beckens von einer P1c-Resektion mit einer Durchtrennung der knöchernen Kontinuität des Beckens.

Die P2-Resektion beinhaltet die Resektion des Azetabulums, entweder intraoder extrakapsulär. Dementsprechend wird bei einer extrakapsulären P2-Resektion auch das proximale Femur reseziert.

Bei der P3-Resektion entfernt man das Os pubis von der Symphyse bis unterhalb des Azetabulums.

Bei einer P4-Resektion wird die Massa lateralis des Os sacrum in die Resektion mit einbezogen.

Abhängig von der Ausbreitung des Tumors im Becken reseziert man die betroffenen Anteile und spricht dann bspw. von einer P1-4-Resektion, wenn man die gesamte Beckenhälfte von der Symphyse bis zur Mittellinie des Os sacrum entfernt oder von einer P2-3-Resektion, wenn man die vorderen Anteile des Beckens mit Azetabulum entfernt.

\section{Operative Durchführung und Rekonstruktionsmöglichkeiten}

Zur Durchführung des operativen Eingriffs wird der Patient in mobiler Seitenlage gelagert, um ein Präparieren sowohl beckeninnenseitig als auch beckenaußenseitig möglich zu machen. Die Hautinzision erstreckt sich nach Enneking von der Symphyse über den Beckenkamm (Crista iliaca) bis, je nach Tumor-

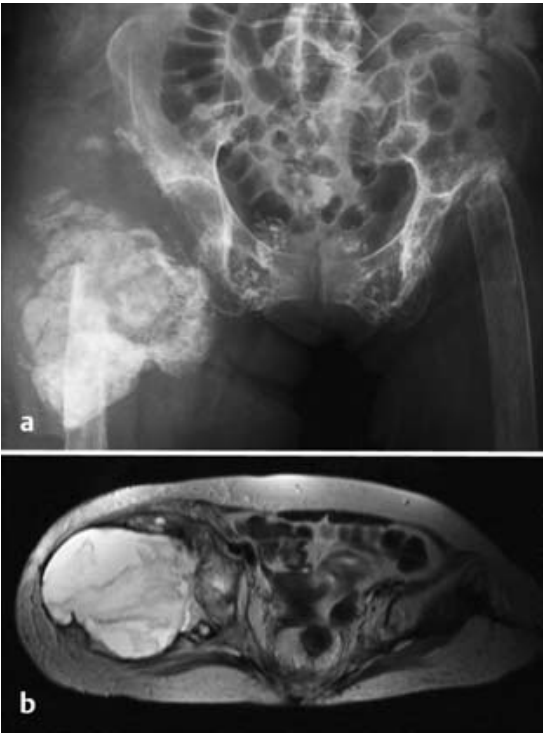

Abb. 2a und b a Sekundäres Osteosarkom, rechter proximaler Femur 10 Jahre nach Strahlentherapie eines Analkarzinoms. Beidseitige Girdlestone-Hüften aufgrund von Strahlennekrosen des Caput femoris. b Eine ausreichende Muskeldeckung (Glutealmuskulatur) ist nicht zu erreichen und die Indikation zur externen Hemipelvektomie gegeben.

ausdehnung, zum Iliosakralgelenk oder den Dornfortsätzen des 5. LWK. Diese Inzision kann bei Bedarf mit einem Hilfsschnitt auf den lateralseitigen Oberschenkel erweitert werden, besonders wenn eine proximale Femurresektion indiziert ist $[5,6]$.

Allen Tumorresektionen ist die Darstellung der Nerven und Gefäße gemeinsam. Besonders die Freipräparation der großen Beckenvenen ist zeitintensiv und kann zu größeren Blutungen mit hämorrhagischem Schock führen. Nervenschädigungen können zu Inkontinenz und Impotenz führen sowie zu ausgeprägten sensomotorischen Ausfällen der betroffenen Seite. Oftmals ist jedoch die Resektion von Nerven oder Nervenwurzeln am Os sacrum Teil des geplanten Eingriffs. Die iatrogene Schädigung von urogenitalen Strukturen oder Darm ist aufgrund der anatomischen Nähe zwischen diesen Strukturen und dem Tumor manchmal schwer zu vermeiden, jedoch auch relativ leicht zu beherrschen.

Eine externe lebensrettende Hemipelvektomie ist bei schwerwiegenden Komplikationen indiziert. Hierüber ist der Patient präoperativ genaustens aufzuklären.

Die Indikation zur geplanten externen Hemipelvektomie ist gegeben, wenn kei- 


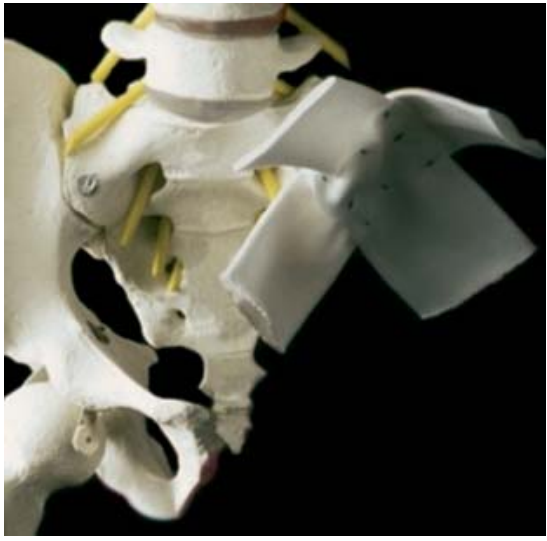

Abb. 3 Hüftverschiebeplastik. Der Hüftkopf wird mittels Mitek-Ankern und zweier Anbindungsschläuche am verbleibenden Becken fixiert.

ne ausreichende Muskeldeckung (Glutealmuskulatur) $\mathrm{zu}$ erreichen ist sowie bei ausgedehnten proximalen Femurtumoren mit Beteiligung des Hüftgelenks (untere Hemipelvektomie, Abb. 2) [1,4].

Sehr selten sind eine Infiltration der Gefäße mit Tumorgewebe oder ein Überschreiten der ISG-Fuge, die eine externe Hemipelvektomie notwendig machen.

Es ist zu bedenken, dass eine externe Hemipelvektomie den Resektionsrand gegenüber einer internen Hemipelvektomie in der Regel nicht verbessert.

Die Möglichkeiten der Rekonstruktion nach interner Hemipelvektomie sind auf wenige funktionelle Möglichkeiten beschränkt.

Die am häufigsten durchgeführte Rekonstruktion ist die Hüftverschiebeplastik, bei der der Hüftkopf mittels Mitek-Ankern und zweier Anbindungsschläuche am verbleibenden Becken fixiert wird (Abb. 3 und 4) [3].

Bei einer zusätzlichen Resektion des proximalen Femurs kann die Hüftverschiebeplastik auch durch Implantation einer Megaprothese des proximalen Femurs erreicht werden (Abb.5). Diese Form der Rekonstruktion ist für P2-, P1-2-, P1-3- und P1-4-Resektionen geeignet.

Die Indikation zur Rekonstruktion mit Beckenteilersatzprothesen muss kritisch gestellt und individuell angepasst werden, da sich oftmals eine erhöhte Infektionsrate zeigte [7].

Eine isolierte P3- sowie eine P1a-Resektion bedürfen keiner Rekonstruktion.
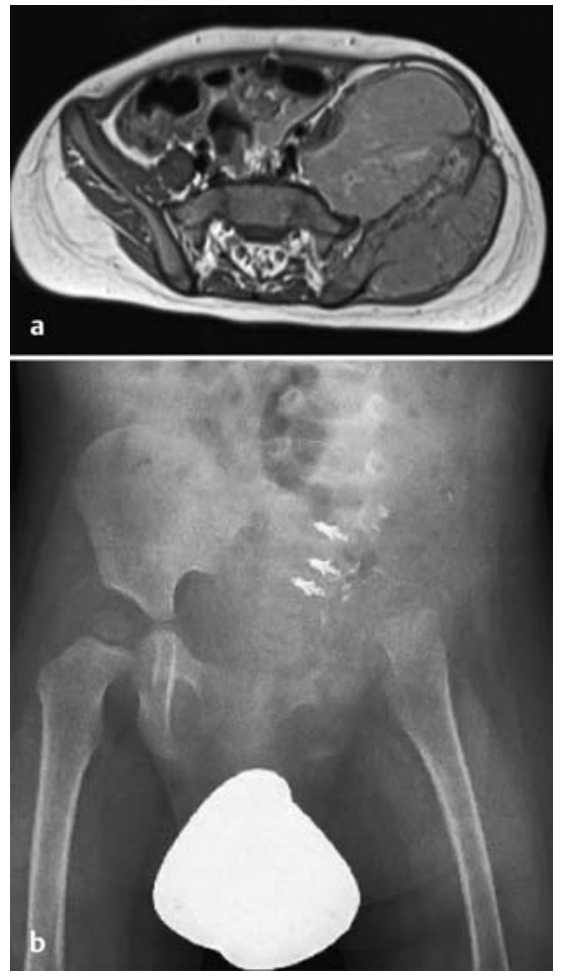

Abb. 4a und $\mathbf{b}$ Beispiel einer P1-2-Resektion eines Ewing-Sarkoms. a Tumor im Bereich des linken Os ilium. b Zustand nach intraartikulärer P1-2-Resektion und Hüftverschiebeplastik.

Bei P1c-Resektionen ist die Rekonstruktion mit Titanstäben, die mit Knochenzement verstärkt werden, und Polyaxialschrauben aus dem Wirbelsäulensystem möglich (Abb. 6).

Dadurch wird zum einen eine Minimierung des Hohlraums erreicht, zum anderen ist durch die Zementapplikation das lokale Einbringen von Antibiotika, die dem Zement zugemischt wurden, möglich. Die Rekonstruktionen mit einem Allograft oder Autograft hat man aufgrund mechanischer Komplikationen weitgehend verlassen.

Bei allen Rekonstruktionsverfahren können postoperativ schwerwiegende Komplikationen auftreten.

Häufig sind massive Wundheilungsstörungen aufgrund von Gefäßresektionen, im Besonderen der A. und V. iliaca interna und der daraus resultierenden Minderversorgung der Glutealmuskulatur, die im postoperativen Verlauf nekrotisch werden kann. Ebenso häufig sind Wundinfektionen aufgrund der großen Wundfläche und der langen OP-Dauer. Diese Komplikationen sind aber beherrschbar, jedoch sind häufige Wundrevisionen so-

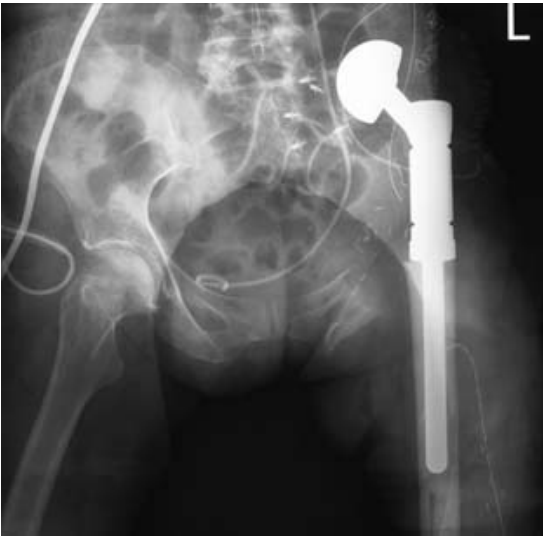

Abb. 5 Hüftverschiebeplastik durch Implantation einer Megaprothese in den proximalen Femur bei extraartikulärer P1-2-Resektion.
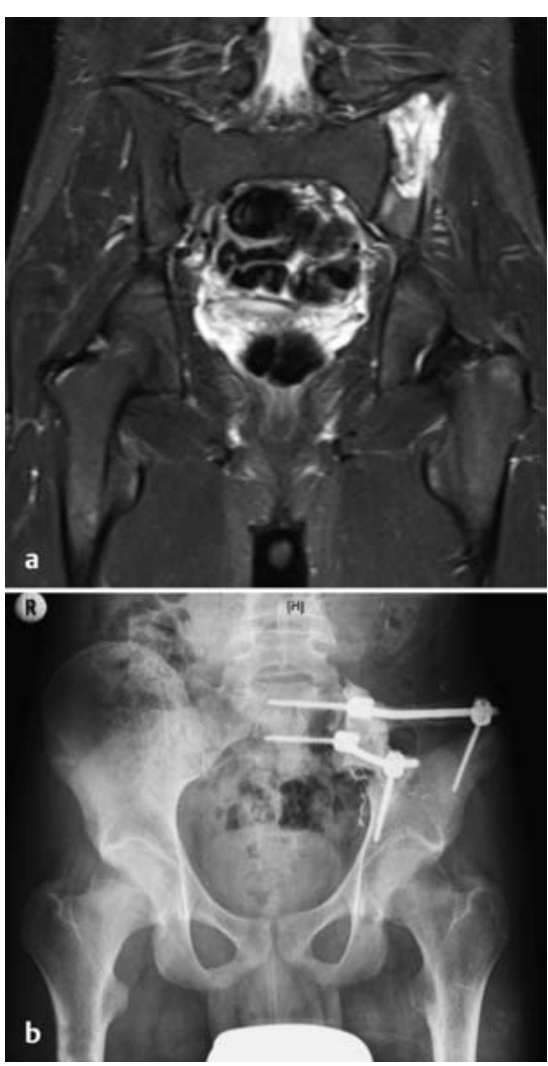

Abb. 6 a und b a Ewing-Sarkom im Os ilium, b P1c-Resektion des lliums und Rekonstruktion mit Titanstäben, die mit Knochenzement verstärkt wurden, und Polyaxialschrauben aus dem Wirbelsäulensystem.

wie die intensive Benutzung von Vakuumverbänden notwendig.

\section{Funktionelle Resultate}

Die funktionellen Resultate bei internen Hemipelvektomien sind überwiegend zufriedenstellend, wenn man den Umfang des chirurgischen Eingriffs mit in Betracht zieht. 
Bei der Hüftverschiebeplastik kommt es zur Beinverkürzung und zum Hinken. Die Benutzung eines Gehstocks ist notwendig, kurze Gangdistanzen können auch ohne Gehstützen bewältigt werden.

Bei P1c-Resektionen ist das funktionelle Resultat oftmals deutlich besser, da das Hüftgelenk erhalten bleibt. Aufgrund der muskulären Resektion besteht auch hier ein Hinken, jedoch ist die Gangfunktion auch ohne Gehstützen deutlich besser.

Die dauerhafte Verwendung eines Rollstuhls ist das Resultat externer Hemipelvektomien. Eine Beckenkorbprothese dient oft nur als Schmuckprothese ohne funktionellen Wert. Jüngere Patienten können mit 2 Unterarmgehstützen ohne Prothese gehfähig werden. Ebenso kann die Anpassung modernen Prothesen die Gehfähigkeit junger Patienten wiederherstellen.

\section{Literatur}

${ }^{1}$ Capanna R, Manfrini M, Pignatti G et al. Hemipelvectomy in malignant neoplasms of the hip region. Ital J Orthop Traumatol 1990; 16: 425-437

2 Enneking WF, Dunham WK. Resection and reconstruction for primary neoplasms involving the innominate bone. J Bone Joint Surg [Am] 1978; 60: 731-746

3 Gebert C, Gosheger G, Winkelmann W. Hip transposition as a universal surgical procedure for periacetabular tumors of the pelvis. J Surg Oncol 2009; 99: 169-172

${ }_{4}$ Karakousis CP. The technique of major amputations for malignant tumors. J Surg Oncol 1983; 23: 43-55

${ }^{5}$ Lackman RD, Crawford EA, Hosalkar HS et al. Internal hemipelvectomy for pelvic sarcomas using a T-incision surgical approach. Clin Orthop Relat Res 2009; 467: 2677-2684

${ }^{6}$ Windhager $R$, Welkerling $H$, Kastner $N$ et al. [Surgical therapy of pelvis and spine in primary malignant bone tumors]. Orthopade 2003; 32: 971-982

7 Witte D, Bernd L, Bruns G et al. Limb-salvage reconstructions with MUTARS hemipelvic endoprosthesis: a prospective multicenter study. Eur J Surg Oncol 2009; 35: 1318-1325
Markus Nottrott, PhD

Funktionsoberarzt

Dr. med. Arne Streitbürger

Oberarzt

Univ.-Prof. Dr. med. Georg Gosheger

Klinikdirektor

Dr. med. Ralf Dieckmann

Assistenzarzt

Dr. med. Marcel-Philipp Henrichs

Assistenzarzt

Prof. Dr. med. Jendrik Hardes

Sektionsleiter Tumor- und

Revisionschirugie

Klinik und Poliklinik für Allgemeine

Orthopädie und Tumororthopädie

Universitätsklinikum Münster

Albert-Schweitzer-Straße 33

48149 Münster

markus.nottrott@ukmuenster.de

Priv.-Doz. Dr. med. Carsten Gebert

Leitender Arzt

Orthopädische Klinik Volmarstein

Lothar-Gau-Straße 11

58300 Wetter 